\title{
Perception of pain after resistance exercise
}

\author{
K F Koltyn, R W Arbogast
}

\begin{abstract}
Objectives-The main objective was to assess the influence of resistance exercise on pain threshold and pain ratings. Secondary objectives included measuring state anxiety, body awareness, systolic blood pressure, diastolic blood pressure, and heart rate responses.

Methods-Pressure (3 kg force) was applied to the middle digit of the left hand for two minutes using a Forgione-Barber pain stimulator before and after (five minute and 15 minutes) resistance exercise and quiet rest. Resistance exercise consisted of 45 minutes of lifting three sets of 10 repetitions at $75 \%$ of an individual's one repetition maximum. Quiet rest consisted of sitting quietly in a room free from distractions.
\end{abstract}

Results-Data were analysed by repeated measures analysis of variance for multifactor experiments. Pain threshold was significantly higher $(p<0.05)$ and pain ratings were significantly lower $(p<0.05)$ five minutes after resistance exercise. Changes in pain perception were accompanied by changes in heart rate, systolic blood pressure, and body awareness after exercise.

Conclusion-A single bout of resistance exercise is capable of modifying the sensation of experimentally induced pain.

(Br F Sports Med 1998;32:20-24)

Keywords: pain perception; resistance exercise; heart rate; blood pressure; body awareness

It has been reported by Raithel ${ }^{1}$ that most pain management programmes prescribe some kind of exercise regimen. Some include aerobic exercise, others include resistance exercise, and still others use a combination of aerobic and resistance exercise. Research has been conducted into whether aerobic exercise, such as cycling and running, is associated with an analgesic effect, ${ }^{2-8}$ and has indicated that aerobic exercise at a sufficient intensity $(>70 \%$ of maximal aerobic capacity) has been associated with increases in pain threshold, ${ }^{4-8}$ as well as decreases in pain ratings. ${ }^{3}$ However, very little research has been conducted on resistance exercise.

In one of the few studies employing resistance exercise, Anshel and Russell ${ }^{9}$ investigated the effects of an aerobic exercise training programme in comparison with a strength training programme on pain perception in men. Forty eight unfit men were randomly assigned to aerobic training, strength training, combined aerobic and strength training, or control conditions. Individuals in the exercise conditions trained three times a week for 12 weeks. Aerobic training consisted of riding a stationary bicycle at an intensity between 60 and $80 \%$ of maximum heart rate. Strength training consisted of lifting 10 repetitions of eight different exercises at $60 \%$ of the individual's 10 repetition maximum. Pain measures included pain tolerance, as well as the individual's subjective appraisal of pain. A football cleat was pressed against the tibialis anterior for lower limb pain tolerance and against the brachioradialis muscle for upper limb pain tolerance. Subjective pain appraisal was measured with the McGill pain questionnaire. Results indicated that 12 weeks of aerobic training significantly increased pain tolerance but only in the upper limb. On the other hand, strength training was not associated with changes in pain tolerance in either the upper or lower limb.

The study by Anshell and Russell ${ }^{9}$ was a training study investigating the chronic effects of exercise on pain. However, little is known about the acute effects-that is, one session - of resistance exercise on pain perception. Can exercise provide immediate relief from pain, and if so, what kinds of exercise are beneficial? It has been shown that one aerobic exercise session is associated with an increase in pain threshold and a decrease in pain ratings. ${ }^{8}$ More research is needed with various forms of exercise. Exercise may serve as an effective and healthy pain management alternative. Thus the primary purpose of this study was to assess the influence of a resistance exercise session on pain threshold and pain ratings. A secondary purpose was to measure blood pressure, heart rate, state anxiety, and body awareness responses associated with exposure to a painful stimulus.

\section{Methods}

SUBJECTS

Thirteen subjects (six women and seven men) with a mean (SD) age of 23 (5) years volunteered to participate in the study. The estimated sample size was computed on the basis of an alpha of 0.05 , a power of 0.80 , and a moderate effect size. ${ }^{10}$ Subjects with previous resistance exercise experience were recruited by notices placed on campus bulletin boards, as well as announcements made in fitness classes. Each volunteer read and signed an informed consent document that had been previously approved by the university's Human Subjects Institutional Review Board.

\section{GENERAL PROCEDURES}

All subjects completed a one repetition maximum test for the following exercises: bench press, leg press, pull downs, and arm extensions. This maximum test was used to calculate
Accepted for publication 31 October 1997 


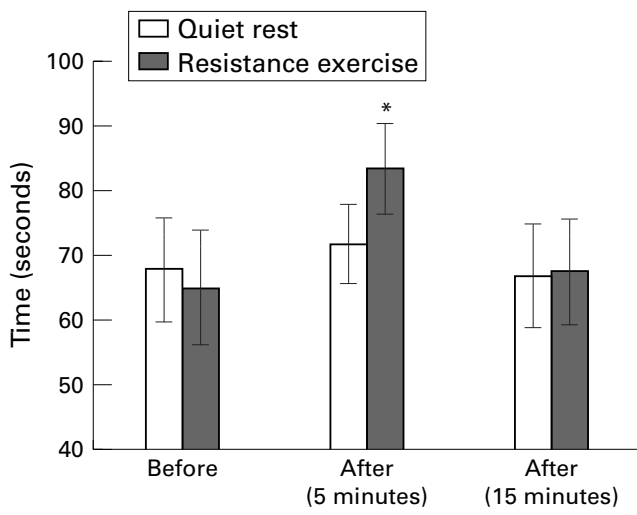

Figure 1 Means and standard errors for pain threshold responses after resistance exercise and quiet rest. ${ }^{\star} p<0.05$ compared with quiet rest.

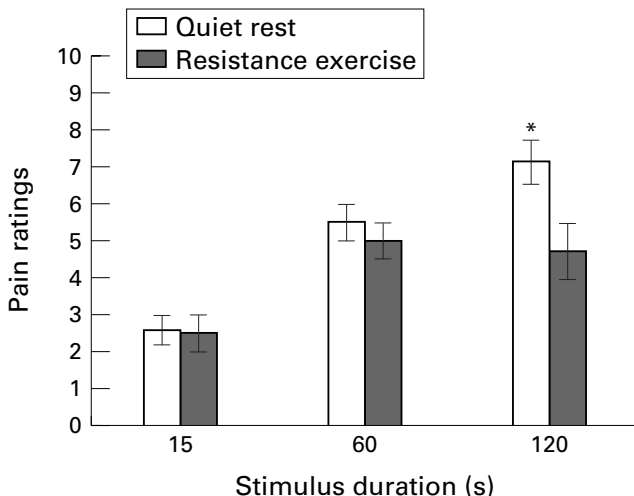

Figure 2 Means and standard errors for pain ratings during the two minutes of exposure to pain after resistance exercise and quiet rest. ${ }^{*} p<0.05$ compared with resistance exercise.

the workload for the exercise condition. Exercise consisted of 45 minutes of lifting three sets of 10 repetitions at $75 \%$ of the individual's one repetition maximum. Each subject completed two randomly assigned conditions: exercise and quiet rest. Quiet rest consisted of 45 minutes of sitting quietly in a room free from distractions. The conditions were completed on separate days at the same time of day.

The pain stimulus consisted of pressure (3 $\mathrm{kg}$ force) being applied to the middle digit of the left hand with a Forgione-Barber pain stimulator. ${ }^{11}$ Subjects inserted the left forefinger into the chute of the pain stimulator where a lucite edge contacted the finger applying $3 \mathrm{~kg}$ of force. Previous research ${ }^{8}$ has shown that this procedure produces a painful sensation but does not cause tissue damage or injury. The pain stimulus was applied to the finger for two minutes before and after (five minutes and 15 minutes) resistance exercise and quiet rest.

Pain threshold and pain ratings were assessed during the two minutes of exposure to pain. Pain threshold was defined as the elapsed time from the initial application of the pain stimulus until the subject perceived the stimulus to be painful. Subjects were instructed to depress a remote push button that was attached to a timer indicating when the stimulus became painful. Pain ratings were given by the subject every 15 seconds during the two minutes of exposure to pain. The subjects were instructed to rate the perception of pain on a ten point category scale. The even numbers of the scale had the following verbal anchors: $0=$ no pain; $2=$ uncomfortable; $4=$ very uncomfortable; 6 $=$ painful; $8=$ very painful; and $10=$ extremely painful.

Blood pressure and heart rate responses were assessed during the two minutes of pain exposure. It has been reported that there is an interaction between blood pressure and pain responsiveness. ${ }^{13-16}$ Blood pressure and heart rate were assessed every 15 seconds during the two minutes exposure with the Ohmeda Finapress blood pressure monitor.

State anxiety and body awareness responses were assessed before and after (10 minutes and 20 minutes) resistance exercise and quiet rest. State anxiety was assessed with Spielberger's state-trait anxiety inventory (STAI). ${ }^{17}$ The STAI has been used routinely in studies investigating the psychological effects of exercise. The STAI has undergone rigorous validation procedures. Body awareness was assessed with the body awareness scale (BAS). ${ }^{18}$ The BAS is a 14 item questionnaire designed to assess somatic aspects of anxiety. Examples of the BAS items are: "My heart is pounding" and "I am aware of my breathing". The BAS was used as part of a continuous validation process.

\section{Results}

Data were analysed with a two (conditions = exercise and quiet rest) $\times$ three (trials $=$ before, 5 minutes after, and 15 minutes after) repeated measures analysis of variance. The GeisserGreenhouse procedure was used to adjust degrees of freedom when the sphericity assumption was not met. The Newman-Keuls procedure was used to probe for the location of mean differences when significant main effects were observed, and a simple effects test was used when significant interactions were observed.

Table 1 Means (SD) for blood pressure and heart rate during the two minute pain exposure before (pre), five minutes after $(P-5)$ and fifteen minutes after $(P-15)$ resistance exercise and quiet rest

\begin{tabular}{|c|c|c|c|c|c|c|c|c|c|}
\hline \multirow[b]{2}{*}{ Variable } & \multicolumn{3}{|l|}{15 seconds } & \multicolumn{3}{|l|}{60 seconds } & \multicolumn{3}{|c|}{120 seconds } \\
\hline & Pre & $P-5$ & $P-15$ & Pre & $P-5$ & $P-15$ & Pre & $P-5$ & $P-15$ \\
\hline \multicolumn{10}{|c|}{ Systolic blood pressure $(\mathrm{mm} \mathrm{Hg})$} \\
\hline Resistance exercise & $111(15)$ & $120(15)$ & $126(15)$ & $130(19)$ & $121(11)$ & $124(15)$ & $137(20)$ & $116(16)$ & $128(20)$ \\
\hline Quiet rest & $100(20)$ & $91(20)$ & $99(20)$ & $115(20)$ & $99(21)$ & $106(25)$ & $114(21)$ & $106(19)$ & $103(24)$ \\
\hline \multicolumn{10}{|c|}{ Diastolic blood pressure $(\mathrm{mm} \mathrm{Hg})$} \\
\hline Resistance exercise & $76(13)$ & $79(9)$ & $87(13)$ & $85(11)$ & $82(8)$ & $96(16)$ & $89(11)$ & $83(17)$ & $95(24)$ \\
\hline Quiet rest & $75(16)$ & $76(17)$ & $84(15)$ & $87(18)$ & $87(18)$ & $89(18)$ & $89(9)$ & $85(12)$ & $95(20)$ \\
\hline \multicolumn{10}{|l|}{ Heart rate (beats/min) } \\
\hline Resistance exercise & $80(14)$ & $100(23)$ & $84(16)$ & $77(13)$ & $101(14)$ & $85(16)$ & $77(5)$ & $104(10)$ & $85(8)$ \\
\hline Quiet rest & $68(9)$ & $61(9)$ & $61(6)$ & $73(7)$ & $66(5)$ & $62(10)$ & $73(8)$ & $68(7)$ & $66(6)$ \\
\hline
\end{tabular}




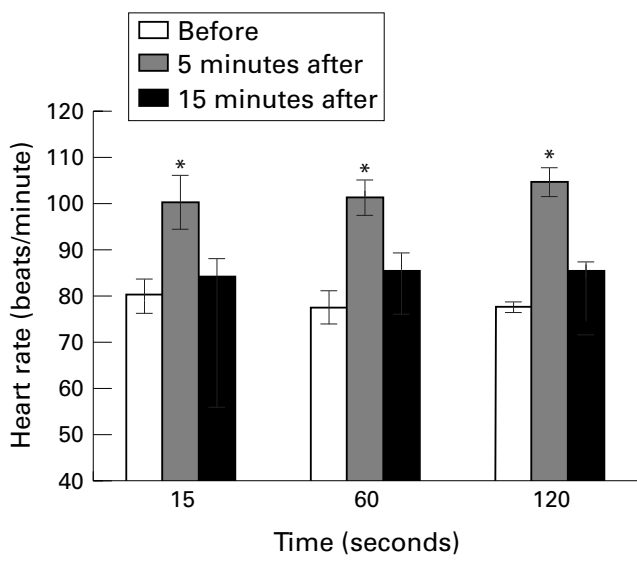

Figure 3 Means and standard errors for heart rate responses before, five minutes and 15 minutes after resistance exercise. ${ }^{\star} p<0.05$ compared with before resistance exercise.

Pain threshold was found to change significantly after resistance exercise. There was a significant conditions by trials interaction $\left(F_{1,12}\right.$ $=7.96 ; \mathrm{p}<0.05)$ for pain threshold. A simple effects post hoc analysis indicated that pain threshold was significantly higher five minutes after the resistance exercise session, in comparison with no change in pain threshold after the quiet rest condition. Figure 1 summarises these results.

Pain ratings during the two minutes pain exposure were found to be different after resistance exercise from those after quiet rest. There was a significant conditions effect $\left(F_{2,10}\right.$ $=6.19 ; \mathrm{p}<0.05)$ for pain ratings. Post hoc analyses showed that pain ratings were significantly lower by the end of the two minutes of pain exposure when assessed about five minutes after the resistance exercise session in comparison with quiet rest (fig 2).

Table 1 summarises the descriptive statistics for heart rate, and systolic and diastolic blood pressure. Heart rate responses during the two minutes of pain exposure were found to vary between the resistance exercise and quiet rest conditions. There was a significant conditions by trials interaction $\left(F_{2,22}=74.22 ; \mathrm{p}<0.001\right)$ for heart rate. Simple effects post hoc analyses indicated that heart rate was significantly higher during the two minutes of pain exposure five minutes after the resistance exercise session than after the quiet rest session, as well as before and 15 minutes after resistance exercise (fig 3).

Systolic blood pressure responses five minutes after resistance exercise appeared to be different from those assessed before and 15 minutes after resistance exercise. These data were analysed by a two (conditions $=$ exercise

Table 2 Means (SD) for state anxiety and body awareness before and after resistance exercise and quiet rest

\begin{tabular}{llll}
\hline Variable & Pre-test & 5 min after & 15 min after \\
\hline State anxiety & $31.3(8)$ & $34.9(10)$ & $31.5(8)$ \\
$\quad$ Resistance exercise & $29.2(6)$ & $31.2(10)$ & $31.5(10)$ \\
$\quad$ Quiet rest & $16.1(3)$ & $19.0(4)^{\star}$ & $16.9(3)$ \\
Body awareness & $16.2(3)$ & $16.7(3)$ & $17.3(4)$ \\
$\quad$ Resistance exercise & & \\
$\quad$ Quiet rest &
\end{tabular}

${ }^{\star} \mathrm{p}<0.05$ compared with pre-test.

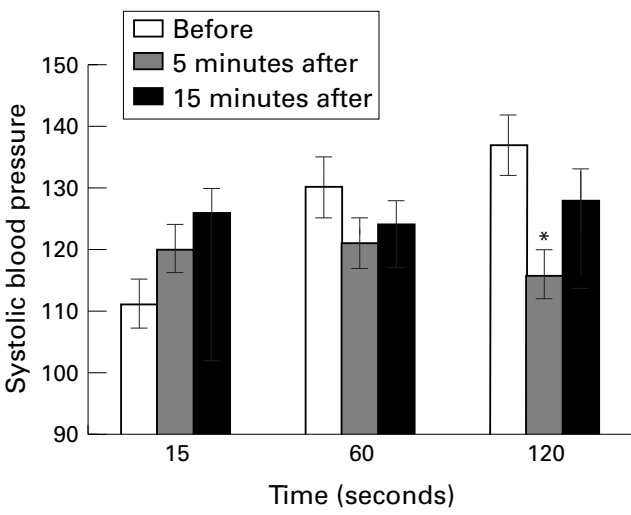

Figure 4 Means and standard errors for systolic blood pressure before, five minutes and 15 minutes after resistance exercise. ${ }^{*} p<0.05$ compared with before resistance exercise.

and quiet rest) $\times$ three (trials $=$ before, five minutes after, 15 minutes after) $\times$ three (time $=$ 15 seconds, 60 seconds, 120 seconds) repeated measures analysis of variance. There was a significant trials $\times$ time interaction $\left(F_{1,3}=21.5\right.$; $\mathrm{p}<0.02)$ for systolic blood pressure. Simple effects post hoc analyses indicated that systolic blood pressure was lower by the end of the two minutes of pain exposure five minutes after the resistance exercise session than 15 minutes after resistance exercise, as well as before resistance exercise (fig 4).

Diastolic blood pressure was found to increase during the two minutes of pain exposure, and there was a significant trials effect $\left(F_{2,10}=4.36 ; \mathrm{p}<0.05\right)$. Post hoc analyses indicated that diastolic blood pressure increased during the two minutes of pain exposure after both resistance exercise and quiet rest. Table 1 summarises the results.

It appears that resistance exercise caused an increase in body awareness as there was a significant conditions by trials interaction $\left(F_{2,24}\right.$ $=12.01 ; \mathrm{p}<0.001)$. It was found through post hoc analyses that body awareness increased significantly five minutes after the resistance exercise session but did not change after quiet rest (table 2). There were no significant conditions, trials, or interaction effects for state anxiety (table 2).

\section{Discussion}

The primary purpose of this investigation was to determine if resistance exercise would be associated with alterations in pain perception. Laboratory studies have found some support for exercise induced analgesic effects of aerobic exercise, ${ }^{19}$ but there has been very little research on resistance exercise. Exercise may serve as an effective, healthy, and less intrusive pain management alternative; however, not all individuals are able to engage in high intensity aerobic exercise. Thus it would seem important to determine whether other forms of exercise are associated with an analgesic effect. Results from this study indicated that pain threshold was significantly increased, in conjunction with lower pain ratings after resistance exercise. These changes in pain perception were evident five minutes after resistance exercise, but by 15 minutes after resistance exercise, pain thresh- 
old had returned to baseline levels. It appears that resistance exercise can alter pain perception, but the duration of this alteration is shorter than that obtained with aerobic exercise. Research with aerobic exercise has found increases in pain threshold during exercise, as well as up to 30 minutes after exercise, ${ }^{4}$ with thresholds returning to baseline levels by one hour after exercise. ${ }^{7}$ Findings from the present study indicate a relatively short analgesic effect after resistance exercise-that is, five minutes. However, we did not assess changes in pain threshold during exercise. If pain threshold was found to change during resistance exercise as it does with aerobic exercise, then the duration of the analgesic effect could potentially increase. Further research is needed to determine the time course of alterations in pain perception during and after resistance exercise. Also, it has been suggested by Jana ${ }^{19}$ that laboratory studies may underestimate the magnitude of the effects of exercise analgesia because of the sterile environment of the laboratory. Other stressors experienced in the field may act synergistically with exercise to have a more profound effect on pain, and this assertion requires further investigation. More research is required in both the field and the laboratory to further our understanding of the effects of different kinds of exercise on pain perception.

A secondary purpose of this study was to assess selected cardiovascular and psychological responses that may interact with pain perception. It has been reported that there is an interaction between cardiovascular and pain regulatory systems. ${ }^{20}$ Exercise significantly alters heart rate and blood pressure responses because of the physiological demands of the activity. In the present study, heart rate and systolic blood pressure responses during the two minutes of pain exposure were found to be significantly different between the exercise and quiet rest conditions. Heart rate was found to be significantly higher during the two minutes of pain exposure five minutes after the resistance exercise condition in comparison with the quiet rest condition. Systolic blood pressure is also elevated after resistance exercise, and it appears that the elevated systolic blood pressure response after exercise was associated with an attenuated increase in blood pressure during the pain exposure. Typically, systolic blood pressure increases during exposure to a painful stimulus, ${ }^{21}$ but in the present study, this increase was attenuated five minutes after resistance exercise. Hoffman and Thore ${ }^{15}$ have reported that once blood pressure is displaced out of the basal range, either by physiological stimuli or pathophysiological states, the endogenous opioid system becomes activated, and endorphins have been linked to the analgesic effect of exercise. Most research into the association between endorphins and the analgesic effect of exercise has used aerobic exercise. Very little is known about whether resistance exercise is associated with an analgesic effect. The results of this study indicate that resistance exercise is associated with a change in pain perception, which is evident within five minutes of the exercise.
Further research is needed to replicate these findings, as well as to determine the mechanism responsible for the analgesic effect of resistance exercise.

Anxiety state did not change significantly after exercise or quiet rest. Other investigators have also not found changes in anxiety after resistance exercise, ${ }^{22-24}$ except for O'Connor et $a l,{ }^{22}$ who did find a significant decrease in anxiety about 90 minutes after resistance exercise performed at $60 \%$ of an individual's 10 repetition maximum. The time course for anxiety reductions after exercise may be different for resistance exercise, and this warrants further attention.

Also, anxiety did not decrease after quiet rest. Several investigators have reported a decrease in anxiety after quiet rest. ${ }^{25}{ }^{26}$ In the present investigation, anxiety did not decrease after quiet rest, and this may be a result of the pain testing. The pain testing could have attenuated the anxiolytic effect that has been previously observed after quiet rest. Another reason why anxiety did not decrease after quiet rest or exercise could be the low anxiety levels of the subjects who volunteered for this study. Anxiety was found to be below the norm, and this may have prevented further decreases from occurring.

Body awareness responses differed from state anxiety responses. It appears that the BAS is very sensitive to somatic changes associated with exercise. Body awareness was found to be significantly elevated after resistance exercise in comparison with quiet rest. Since the BAS was designed to tap into more of a somatic component of anxiety, this elevation could indicate that the individuals were more aware of somatic sensations after resistance exercise than after quiet rest. This awareness of more salient signals-for example, sweating and heart pounding - may have diverted attention away from the pain stimulus, and it has been reported that distraction can significantly alter pain perception. ${ }^{27}{ }^{28} \mathrm{It}$ is possible that exercise did not have an analgesic effect, but rather attention was diverted away from the pain stimulus. Further research into this possibility is needed.

In summary, it appears that one resistance exercise session performed at an intensity of $75 \%$ of one repetition maximum is associated with changes in pain perception that are not evident after quiet rest. Specifically, these changes include an increase in pain threshold accompanied by lower pain ratings after the resistance exercise session. These changes in pain perception are in conjunction with changes in heart rate, systolic blood pressure, and body awareness responses. It is concluded that a single bout of resistance exercise is capable of modifying the sensation of experimentally induced pain.

This work was supported by a Research Opportunity Grant from the University of North Texas.

1 Raithel KS. Chronic pain and exercise therapy. Physician and Sportsmedicine 1989;17:203-10.

2 Haier RJ, Quaid K, Miller JS. Naloxone alters pain perception after jogging. Psychiatry Res 1981;5:231-2. 
3 Janal MN, Colt EW, Clark WC, Glusman M. Pain sensitivity, mood and plasma endocrine levels in man following long-dista $13-25$.

4 Pertovaara A, Huopaniemi T, Virtanen A, Johansson G. The influence of exercise on dental pain thresholds and the release of stress hormones. Physiol Behav 1984;33:923-6.

5 Kemppainen P, Pertovaara A, Huopaniemi T, et al. Modification of dental pain and cutaneous thermal sensitivity by physical exercise in man. Brain Res 1985;360:33-44.

6 Olausson B, Eriksson E, Ellmarker L, et al. Effects of naloxone on dental pain threshold following exercise and low frequency transcutaneous nerve stimulation: a comparative study in man. Acta Physiol Scand 1986;126:299305.

7 Droste C, Greenlee MW, Schreck M, Roskam H. Experimental pain thresholds and plasma beta-endorphin levels mental pain thresholds and plasma beta-endorphin

8 Koltyn KF, Wertz AL, Gardiner RL, Nelson TF. Perception of pain following aerobic exercise. Med Sci Sports Exerc of pain following

9 Anshel MH, Russell KG. Effect of aerobic and strength training on pain tolerance, pain appraisal and mood of unfit males as a function of pain location. I Sports $\mathrm{Sc}$ 1994;12:535-47

10 Kraemer HC, Thieman S. How many subjects? Newbury Park, CA: Sage, 1987

11 Forgione AG, Barber TX. A strain gauge stimulator. Psychophysiology 1971;8:102-6.

12 Morgan WP, Horstman DH. Psychometric correlates of pain perception. Percept Mot Skills 1978;47:27-39.

13 Bruehl S, Carlson CR, McCubbin JA. The relationship between pain sensitivity and blood pressure in normotensives. Pain 1992;48:463-7.

14 Friedman R, Murphey D, Persons W, McCaughran JA. Genetic predisposition to hypertension, elevated blood pressure and pain sensitivity: a functional analysis. Behav Brain Res 1984;12:75-9.

15 Hoffman P, Thoren P. Electric muscle stimulation in the hind leg of the spontaneously hypertensive rat induces a long-lasting fall in blood pressure. Acta Physiol Scand 1988;
133:211-19.

6 Zamir N, Shuber E. Altered pain perception in hypertensive humans. Brain Res 1980;201:471-4.

17 Spielberger CD, Gorsuch RL, Lushene RE, et al. Manual for the state-trait anxiety inventory. Palo Alto, CA: Consulting Psychologists Press, 1983.

18 Wang Y, Morgan WP. Convergent validity of a body awareness scale. Med Sci Sports Exerc 1991;23:S42.

19 Janal MN. Pain sensitivity, exercise and stoicism. $f R$ Soc Med 1996;89:376-81.

20 Randich A, Maixner W. Interactions between cardiovascular and pain regulatory systems. Neurosci Biobehav Rev 1984;8: 343-67.

21 Hilgard ER, Morgan AR. Heart rate and blood pressure in the study of laboratory pain in man under normal conditions and as influenced by hypnosis. Acta Neurobiol Exp (Warsz) 1975;35:741-59.

22 O'Connor PJ, Bryant CX, Veltri JP, Gebhardt SM. State anxiety and ambulatory blood pressure following resistance exercise in females. Med Sci Sports Exerc 1993;25:516-21.

23 Raglin JS, Turner PE, Eksten F. State anxiety and blood pressure following 30-min of leg ergometry or weight training. Med Sci Sports Exerc 1993;25:1044-8.

24 Koltyn KF, Raglin JS, O’Connor PJ, Morgan WP. Influence of weight training on state anxiety, body awareness and blood pressure. Int f Sports Med 1995;16:266-9.

25 Bahrke MS, Morgan WP. Anxiety reduction following exercise and meditation. Cognitive Therapy and Research 1978;2:323-3.

26 Brown DR, Morgan WP, Raglin JS. Effects of exercise and rest on the state anxiety and blood pressure of physically challenged college students. $\mathcal{F}$ Sports Med Phys Fitness 1993;33:300-5.

27 Fillingim RB, Roth DL, Haley, WE. The effects of distraction on the perception of exercise-induced symptoms. F Psychosom Res 1989;33:241-8.

$28 \mathrm{McCaul} \mathrm{KD}$, Malott JM. Distraction and coping with pain. Psychol Bull 1984;95:516-33.

\section{British Fournal of Sports Medicine http://www.bjsportmed.com}

Visitors to the world wide web can now access the British fournal of Sports Medicine either through the BMJ Publishing Group's home page (http://www.bmjpg.com) or directly by using its individual URL (http://www.bjsportmed.com). There they will find the following:

- Current contents list for the journal

- Contents lists of previous issues

- Members of the editorial board

- Information for subscribers

- Instructions for authors

- Details of reprint services.

A hotlink gives access to:

- BMJ Publishing Group home page

- British Medical Association web site

- Online books catalogue

- BMJ Publishing Group books.

The web site is at a preliminary stage and there are plans to develop it into a more sophisticated site. Suggestions from visitors about features they would like to see are welcomed. They can be left via the opening page of the BMJ Publishing Group site or, alternatively, via the journal page, through "about this site". 